\title{
Transcranial Doppler ultrasound findings in children with moderate-to-severe traumatic brain injury following abusive head trauma
}

\author{
Marlina E. Lovett ${ }^{1,2}$ (D) $\cdot$ Tensing Maa ${ }^{1,2}$ (D) $\cdot$ Melissa Moore-Clingenpeel ${ }^{3}$ (D) $\cdot$ Nicole F. O'Brien $^{1,2}$ \\ Received: 8 April 2019 / Accepted: 1 November 2019 / Published online: 28 November 2019 \\ (C) Springer-Verlag GmbH Germany, part of Springer Nature 2019
}

\begin{abstract}
Purpose Abusive head trauma (AHT) is the leading cause of fatal head injuries for children under 2 years. The objective was to evaluate, using transcranial Doppler ultrasound (TCD), whether children with AHT have a similar neurovascular response to injury compared with children without AHT.

Methods Retrospective sub-analysis of previously prospectively acquired data in a pediatric intensive care unit in a level 1 trauma hospital. TCD was performed daily until hospital day 8, discharge, or death. Neurologic outcome was assessed using the Glasgow Outcome Scale Extended (GOS-E Peds) at 1 month from initial injury.

Results Sixty-nine children aged 1 day to 17 years with moderate-to-severe traumatic brain injury were enrolled. Fifteen children suffered AHT and 54 had no suspicion for AHT. Fifteen children with AHT underwent 80 serial TCD examinations; 54 children without AHT underwent 308 exams. After standardization for age and gender normative values, there was no statistically significant difference in mean cerebral blood flow velocity of the middle cerebral artery $\left(V_{\mathrm{MCA}}\right)$ between children with and without AHT. There was no difference in the incidence of extreme cerebral blood flow velocity (CBFV, greater or less than 2 standard deviations from normative value) between groups. Within the AHT group, there were no statistically significant differences in $V_{\mathrm{MCA}}$ between children with a favorable (GOS-E Peds 1-4) versus unfavorable neurologic outcome (GOS-E Peds 5-8).

Conclusion Children with AHT have no significant differences in $V_{\mathrm{MCA}}$ or percentage of extreme CBFV in the middle cerebral artery compared to with those without AHT.
\end{abstract}

Keywords Transcranial Doppler ultrasound · Traumatic brain injury · Abusive head trauma

\section{Introduction}

Abusive head trauma (AHT) is a leading cause of death in children under 2 years [6]. The Centers for Disease Control and Prevention defines AHT as injury to the skull or

Work was performed at: Nationwide Children's Hospital, Columbus, $\mathrm{OH}$.

Marlina E. Lovett

marlina.lovett@nationwidechildrens.org

1 Division of Critical Care Medicine, Nationwide Children's Hospital, 700 Children's Dr., Columbus, OH 43205, USA

2 Department of Pediatrics, The Ohio State University, Columbus, $\mathrm{OH}$, USA

3 Biostatistics Core, Research Institute at Nationwide Children's Hospital, Columbus, OH, USA intracranial contents of children less than 5 years of age, due to inflicted blunt impact and/or violent shaking, with specific exclusion of unintentional injuries [19]. Compared with children who suffer non-abusive head injury, children with AHT have higher rates of death and morbidity $[4,7]$.

Mechanisms of injury in children suffering AHT can be both impulsive ("non-impact forces generated by alternating angular acceleration and deceleration of the cranial vault") and impact-loading forces ("direct application of force to the head") [24]. These forces may result in various patterns of brain injury, which often co-exist in the same child: axonal injury, parenchymal injury, skull fractures, and extra-axial hemorrhage $[6,14,24]$. Children with AHT are at risk for hypoxic-ischemic injury due to vascular compromise or asphyxia [18]. Furthermore, children with AHT may often be exposed to repeated injury over time and may have acute, subacute, and chronic injury at the time of presentation. 
Therefore, given the multiple potential contributors to injury in AHT, it remains unclear if the neurovascular response, including changes in cerebral blood flow velocity (CBFV) as well as cerebral autoregulation, is different in children with AHT compared with other forms of traumatic brain injury (TBI).

Transcranial Doppler ultrasonography (TCD) uses lowfrequency ultrasound to measure $\mathrm{CBFV}$ in the vessels within the circle of Willis. This non-invasive modality does not expose the patient to radiation, can be performed at the bedside, allows for evaluation of cerebral hemodynamics in real-time and for repeated measurement. Following severe TBI, cerebral blood flow may be dysregulated, often having an initial period of hypoperfusion followed by hyperemia [22]. Studies using TCD in children with severe TBI have shown that abnormal CBFV may be associated with poor outcome [11]. However, many studies exclude children with AHT, as the mechanism and resultant central nervous system injuries may be different. As such, there is a paucity of literature describing the neurovascular response in children with AHT.

Therefore, we aimed to use TCD to evaluate the CBFV in children suffering AHT. We hypothesized that children with AHT would have higher rates of extreme CBFV (defined as greater or less than 2 standard deviations from normative values) and unfavorable neurologic outcome compared with children with non-abusive head trauma. We further hypothesized that within the cohort of children with AHT, those with unfavorable neurologic outcomes would have higher rates of extreme CBFV compared with those with favorable outcome.

\section{Methods}

This retrospective sub-analysis was performed on data previously prospectively acquired as a study of children with TBI that was performed at a level 1 pediatric trauma center [17]. The parent study was approved by the institutional review board and informed consent was obtained prior to patient enrollment. Children 1 day to 17 years of age, who were admitted to the pediatric intensive care unit (PICU) with a diagnosis of moderate or severe TBI, with abnormal head imaging, were eligible for inclusion. Children were excluded if they were deemed to have a non-survivable injury with a Glasgow coma score (GCS) of 3T with fixed and dilated pupils, and death was imminent.

Demographic data were extracted from the medical record, including age, gender, mechanism of injury, injury severity score (ISS), and post-resuscitation GCS [9]. Head imaging, laboratory data, surgical intervention, and the medication administration record were reviewed.

All children with TBI received similar care. Children with severe TBI were treated according to severe TBI management guidelines [10]. Neurosurgical intervention, including mass lesion resection, a primary decompressive craniectomy, or placement of an intracranial pressure monitor was at the discretion of the neurosurgical team. All children with suspicion for AHT underwent skeletal survey, ophthalmologic examination, and assessment by a child abuse expert. Rotterdam and Marshall scores were calculated based upon initial head imaging read by a pediatric radiologist.

\section{Transcranial Doppler ultrasonography}

TCD was performed at the patient's bedside by one of two experienced sonographers using a commercially available TCD ultrasonography unit (Sonara Digital TCD; Care Fusion, Middleton, WI) with a 2-MHz transducer. Initial TCD was performed within $24 \mathrm{~h}$ of injury and daily thereafter until hospital day 8 , discharge, or death, whichever occurred first. The middle cerebral arteries (MCAs) and basilar artery (BA) were insonated at 2-mm intervals using previously described methods [1,12]. The depth and angle of insonation giving the highest mean flow velocity and best waveform was chosen. The mean cerebral blood flow velocity $\left(V_{\mathrm{MCA}}\right)$ was recorded on both the right and left side. The bilateral $V_{\mathrm{MCA}}$ data was then averaged for one composite value. The pulsatility index, calculated on the TCD unit using the equation: $\left(V_{\text {systolic }}-V_{\text {diastolic }}\right) / V_{\mathrm{MCA}}$ was noted. TCD was only performed when no changes to the ventilator or interventions to maintain cerebral perfusion pressure had occurred within the previous hour. Physicians caring for these children were blinded to TCD findings.

Since $\mathrm{CBFV}$ varies with age and gender, $V_{\mathrm{MCA}}$ was reported as standard deviations from previously published normative values for children of similar age and gender. Intubated patients were compared with critically ill, intubated, and sedated children without neurologic illness or injury; after extubation, patients were compared with healthy children of similar age and gender $[3,16]$. Children with $V_{\mathrm{MCA}}$ greater or less than 2 standard deviations from their age and gender normative values were labeled as having extreme CBFV.

Cerebral autoregulation was investigated using the transient hyperemic response ratio (THRR), a dynamic method of autoregulation testing that involves a direct challenge to the cerebral vasculature [20]. Based on previously published values for critically ill children without neurologic illness or injury, a THRR $\geq 1.035$ was defined as intact whereas a value $<1.035$ was non-intact cerebral autoregulation [13].

\section{Outcome}

Short-term neurologic outcome was assessed using the Glasgow Outcome Scale Extended Pediatric Version (GOSE Peds) 1 month from the initial injury [2]. The GOS-E Peds is 
Table 1 Demographic, clinical, and radiographic characteristics of all children with moderate-tosevere TBI who underwent transcranial Doppler ultrasound. Medians with interquartile ranges are shown when applicable

\begin{tabular}{lllc}
\hline & AHT $(n=15)$ & Non-AHT $(n=54)$ & $p$ value \\
\hline Age (years) & $0.33(0.24,0.58)$ & $10.2(4.52,12.85)$ & $<0.0001$ \\
ICP monitors & $4(20 \%)$ & $20(37 \%)$ & 0.55 \\
GCS & $9(6,10.5)$ & $9(7,9.75)$ & 0.76 \\
Gender & $80 \%$ male & $70.4 \%$ male & 0.53 \\
Race & $20 \%$ female & $29.6 \%$ female & \\
& Caucasian: $73 \%$ & Caucasian: $70.4 \%$ & \\
& African American: $27 \%$ & African American: $24 \%$ & \\
& Hispanic: $0 \%$ & Hispanic: $3.7 \%$ & 0.33 \\
Marshall & Asian: $0 \%$ & Asian: $0 \%$ & 0.45 \\
Rotterdam & Other: $0 \%$ & Other: $1.9 \%$ & 0.25 \\
ISS & $3(2.5,6)$ & $3(2,5)$ & $<0.0001$ \\
Seizures & $3(2,3.5)$ & $2(2,3)$ & $>0.99$ \\
Cardiac arrest & $16(11.5,28.3)$ & $22(15.5,26.5)$ & 0.47 \\
PICU LOS (days) & $11(73.3 \%)$ & $2(3.7 \%)$ & 0.58 \\
GOS-E Peds & $1(6.7 \%)$ & $3(5.5 \%)$ & 0.53 \\
Mortality rate & $6(4,7)$ & $6(4,9)$ & $3(1,5)$ \\
\hline
\end{tabular}

$T B I$, traumatic brain injury; $A H T$, abusive head trauma; non-AHT, non-abusive head trauma; $I C P$, intracranial pressure monitor; GCS, Glasgow Coma Score; ISS, injury severity score; $P I C U$, pediatric intensive care unit; $L O S$, length of stay a validated measure of short-term neurologic outcome (typically performed at 3 or 6 months following injury) and classifies patients into: 1) upper good recovery, 2) lower good recovery, 3) upper moderate disability, 4) lower moderate disability, 5) upper severe disability, 6) lower severe disability, 7) vegetative state, and 8) death. A priori outcomes were dichotomized into those with a favorable outcome (1-4) and unfavorable neurologic outcome (5-8).

\section{Statistical analysis}

Initial data analysis was performed with the entire cohort and further subdivided by TBI severity (moderate or severe) and presence or absence of a diagnosis of AHT. Patients with AHT were further analyzed based on neurologic outcome, dichotomized as favorable neurologic outcome versus unfavorable neurologic outcome. Descriptive continuous data were reported as median with first and third quartiles, and categorical data were reported as percentage. Univariate analysis was performed using the Mann-Whitney for continuous variables and Fisher's Exact for categorical variables. Comparison of the partial pressure of carbon dioxide, hematocrit, hyperthermia incidence, incidence of extreme blood flow velocity, and intact cerebral autoregulation over time between groups was performed using linear and binomial mixed effects models, with Holm-Sidak corrections for multiple comparisons where warranted. For all mixed effects models, a group $\times$ time interaction was first evaluated. Where the interaction was deemed probable $(p<0.10)$, daily group differences were tested; when the interaction $p$ value was $>0.10$, average values over time were compared between groups. Statistical significance for all other tests was assumed with $p \leq 0.05$. Statistical analysis was performed using GraphPad Prism 7, SAS 9.4, or Microsoft Excel.

\section{Results}

Sixty-nine children with moderate-to-severe TBI were included. Fifteen children suffered AHT, and 54 had no suspicion of AHT. Demographic and clinical data are present in Tables 1 and 2. The median initial GCS was 9 in both groups. Nearly half of children in both groups had severe TBI (47\% with AHT and $48 \%$ without). Within the AHT group, all patients had a component of subdural hemorrhage, 13/15 (87\%) had retinal hemorrhages, 3/15 (20\%) had rib fractures, $2 / 15(13 \%)$ had a liver laceration, 2/15 (13\%) had vertebral fractures, 1/15 (6.7\%) had an ulnar fracture, and 1/15 (6.7\%) had spinal cord transection. Most (93\%) children with AHT underwent electroencephalogram monitoring, 4 had clinical seizures alone and 7 had both clinical and subclinical seizures. Seventythree percent of children with AHT experienced at least one seizure whereas only $3 \%$ of those with non-AHT had a seizure $(p<0.0001)$. The median GOS-E Peds for children without 


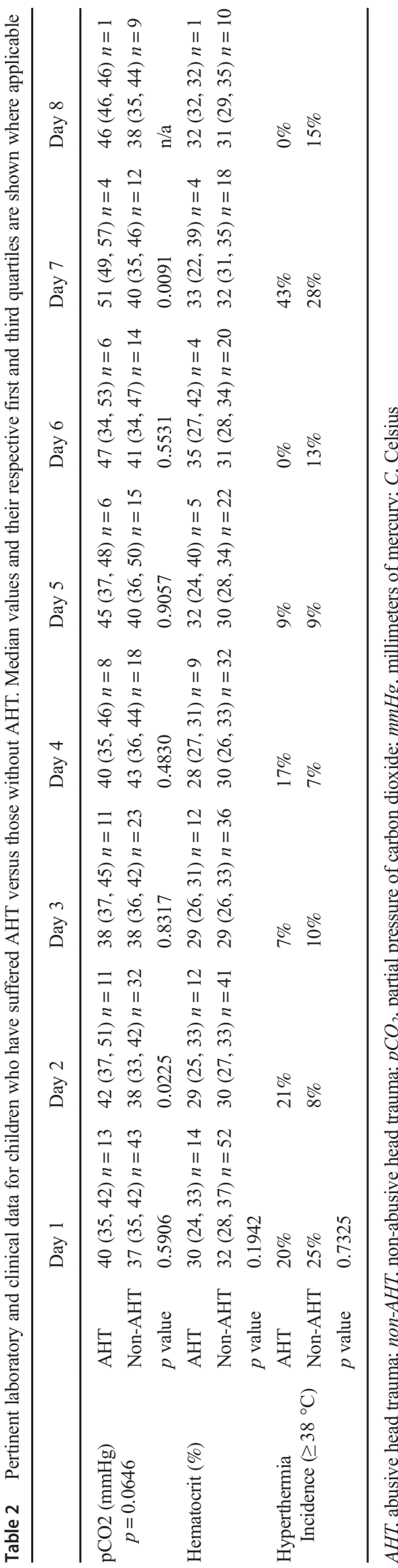

AHT was $3(1,5)$ compared with $5(1,5)$ in those with AHT $(p=0.58)$.

Eighty serial TCD studies were performed in the AHT group, with an average of 5.8 studies/patient. Three hundred six studies were performed in children without AHT, with an average of 5.7 studies/patient. After standardization for gender and age normative values, using a mixed effects model, there was no significant difference in $V_{\mathrm{MCA}}$ for children with AHT versus those with non-AHT on a daily basis $(p=0.9265)$ or averaged across time $(p=0.3405)$ (Fig. 1). There was no significant difference in the percentage of extreme CBFV between groups (Table 3 ). There was no significant daily difference in the pulsatility index of the MCA between groups ( $p=$ 0.243 ), nor was there a difference when averaged across time $(p=0.1347)$. Basilar artery flow was not different between groups $(p=0.6245)$ when all data points were combined nor when averaged across time $(p=0.5335)$.

The initial analysis was performed with both moderate and severe TBI patients combined. Further analysis of children with severe TBI ( 7 children in the AHT group and 26 in the non-AHT group) revealed no statistically significant daily differences in $V_{\text {MCA }}$ between groups ( $p$ value $=0.9683$ ), nor a significant difference when values were averaged over time $(p=0.9390)$. However, $21 \%$ of studies in the AHT group had extreme CBFV versus $37 \%$ in the non-AHT group $(p=0.2020$, Fig. 2$)$. Analysis of children with moderate TBI (AHT versus non-AHT) showed no significant differences $(p=0.7978)$ nor when values were averaged over time $(p=0.1778)$. There was no statistically significant difference between groups when evaluating the incidence of extreme CBFV (30\% in AHT and 30\% in non-AHT moderate TBI groups, $p=0.7884$ ).

In children with AHT, there were no significant differences between the standardized $V_{\mathrm{MCA}}$ in children with a favorable versus unfavorable neurologic outcome $(p=0.3509)$. Furthermore, there was no significant difference in the percentage of extreme CBFV $(p=0.8748)$. There was no significant difference in basilar artery flow velocity between groups $(p=0.7012$ ).

When evaluating all TCD studies, $43 \%$ of studies with AHT versus $53 \%$ of studies in children without AHT had intact cerebral autoregulation based upon the THRR $(p=$ 0.4345 , Table 4). When evaluating children with AHT by neurologic outcome, there was no difference in the total percentage of studies with intact autoregulation (48\% of favorable studies versus $37 \%$ of unfavorable studies had intact autoregulation, $p=0.5607$ ).

\section{Discussion}

Abusive head trauma kills children worldwide. In the USA wq21 alone, there is a $20 \%$ mortality rate for children under 2 years of age [24]. Survivors of AHT are often left with significant morbidity, including motor deficits, visual impairment, 
Fig. $1 V_{\mathrm{MCA}}$ in children with and without abusive head injury. Abbreviations: AHT, abusive head trauma; $V_{\mathrm{MCA}}$, mean cerebral blood flow velocity in the middle cerebral artery

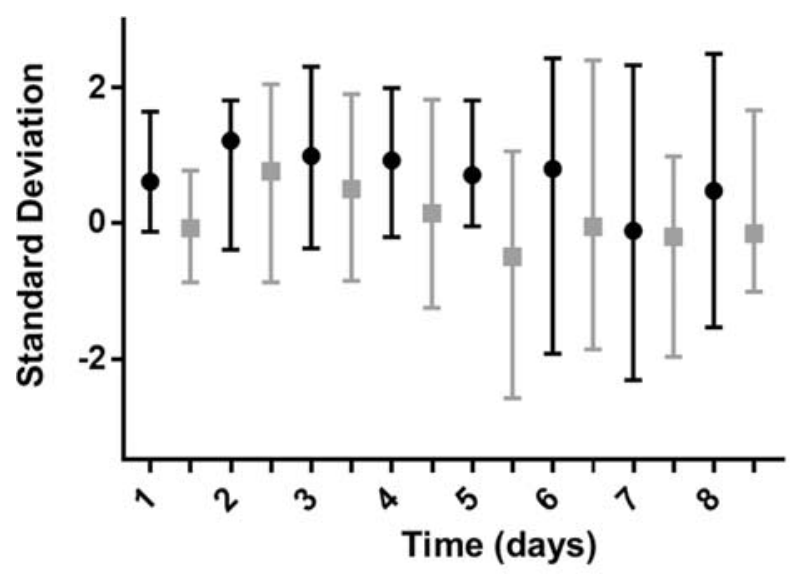

- $\mathrm{AHT}$ - Non-AHT

Abbreviations: $V_{M C A}$ - mean cerebral blood flow velocity in the middle cerebral artery, AHT - abusive head rauma; non-AHT - non-abusive head trauma

cognitive deficits, speech impairment, seizures, and behavioral disorders [15]. In a cohort of 940 children with AHT, 72\% had significant long-term disability 5 years after injury [15]. Given the significant impact on both mortality and long-term functioning, it is imperative that clinicians gain a better understanding of the acute cerebral hemodynamics following AHT to evaluate for potential treatment opportunities.

Transcranial Doppler ultrasound is one modality that provides a non-invasive window into the brain's cerebral hemodynamics. This modality may be helpful to elucidate changes, particularly when there are no invasive modalities used or available. To our knowledge, this is the first reported study to evaluate TCD parameters in a cohort of children with AHT. When evaluating $V_{\mathrm{MCA}}$ in children with and without AHT, there was no significant difference in values standardized for age and gender nor in the percentage of extreme CBFV between groups. While not statistically significant, when analyzed by severity of TBI, we did see that within the severe TBI group, children with AHT had lower rates of extreme CBFV compared with the non-AHT cohort. This is in contrast to our hypothesis and warrants further investigation. Children at risk for repetitive injury may have less of a hyperemic response to an acute traumatic event. In a mouse model of repetitive closed-head injury (once daily for 5 days), cerebral blood flow was reduced following injury and did not return to baseline for $72 \mathrm{~h} \mathrm{[5].} \mathrm{It} \mathrm{is} \mathrm{unclear} \mathrm{how} \mathrm{these} \mathrm{findings} \mathrm{translate}$ to humans, but if similar findings are present, this may be a preliminary explanation for the slight differences seen in extreme CBFV within the severe TBI group.
We aimed to identify if specific transcranial Doppler ultrasound findings were associated with neurologic outcome in children with AHT. There were no statistically significant differences seen in either median $V_{\mathrm{MCA}}$ or the percentage of extreme CBFV between children with AHT and a favorable or unfavorable neurologic outcome. This is in contrast to prior adult studies of severe TBI patients in which patients with normal TCD data had lower rates of death and higher rates of a good or normal neurologic outcome compared with patients with either hypoperfusion or vasospasm [23, 25]. However, the number of children within our cohort suffering AHT was small, and our cohort included both moderate and severe TBI.

Similar to the $V_{\mathrm{MCA}}$ data, there were no significant differences in the percentage of studies with intact autoregulation between children with or without AHT, or within the AHT group dichotomized by neurologic outcome. This is in contrast to a prior adult study of closed-head injury where those with unfavorable outcomes had lower THRRs than those with favorable outcomes [21]. However, we evaluated autoregulation as a categorical variable based on the presence or absence of intact autoregulation. In addition, we investigated autoregulation solely once per day, whereas continuous autoregulation monitoring may provide further insight to the brain's autoregulatory response on an hour by hour basis.

When evaluating our data, it is worth mentioning that within our cohort, no child had documented suspicion for prior abusive injury. Therefore, it remains unclear as to whether our cohort presented with solely acute or acute on chronic
Table 3 Percentage of studies with extreme blood flow velocity for children with abusive versus non-abusive head injury

\begin{tabular}{lcccccccc}
\hline & Day 1 & Day 2 & Day 3 & Day 4 & Day 5 & Day 6 & Day 7 & Day 8 \\
\hline AHT & $13.3 \%$ & $28.6 \%$ & $28.6 \%$ & $25 \%$ & $20 \%$ & $28.6 \%$ & $50 \%$ & $50 \%$ \\
Non-AHT & $15.7 \%$ & $32.7 \%$ & $28.6 \%$ & $35.7 \%$ & $47.2 \%$ & $51.5 \%$ & $38.5 \%$ & $35.3 \%$ \\
$p$ value & 0.2783 & & & & & & & \\
\hline
\end{tabular}

$A H T$, abusive head trauma; non-AHT, non-abusive head trauma 
Fig. 2 Extreme CBFV in severe TBI only. Abbreviations: AHT, abusive head trauma, CBFV, cerebral blood flow velocity, TBI, traumatic brain injury

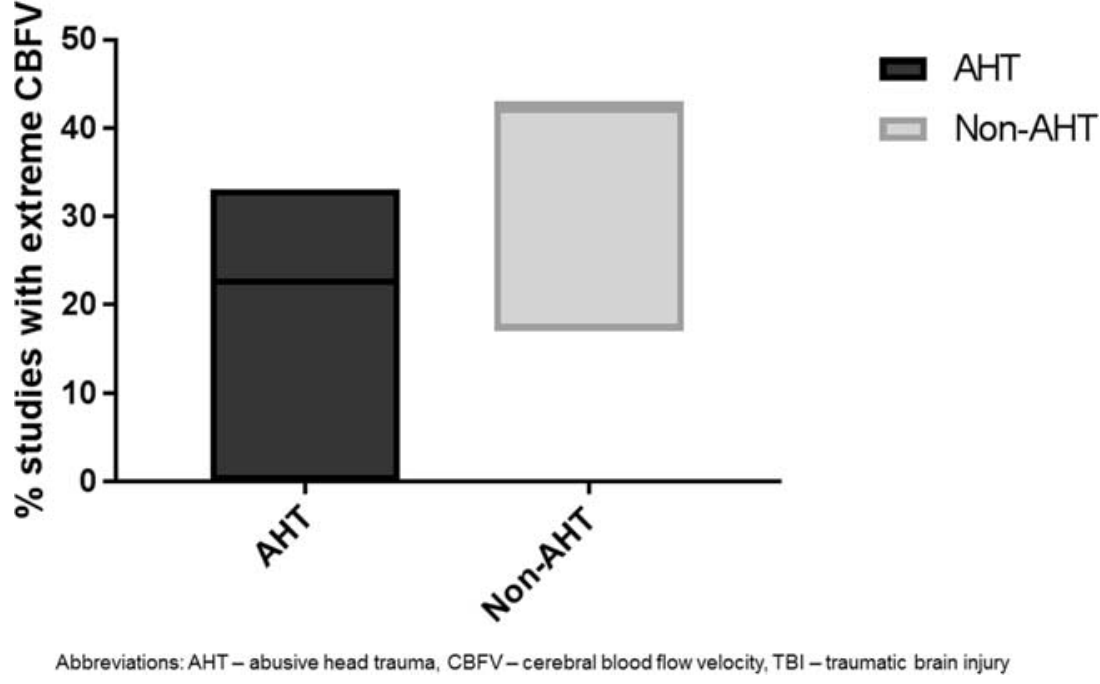

head injury. It is unknown whether children with chronic AHT would have similar findings to our cohort. This is an inherent limitation of the field as often when there is suspicion for abuse, interventions are taken to place the child in a safe environment. It may be possible to begin to understand the cerebral hemodynamics of chronic head injury by comparing $V_{\mathrm{MCA}}$ in children with acute subdural hemorrhage with those with evidence of both acute and chronic subdural hemorrhage on initial imaging. Children at risk for repetitive head injury, such as athletes with repeated concussions, may serve as an alternative population in which one could further elucidate the longitudinal TCD changes attributed to chronic head injury.

In general, the results of this study were in contrast to our hypothesis. When combining THRR and $V_{\text {MCA }}$ data, this study suggests that children with AHT have a similar neurovascular response to trauma, as measured by TCD, to children without AHT. However, our study evaluated a cohort of children with both moderate and severe TBIs. To better inform the literature regarding changes in transcranial Doppler ultrasound of children with AHT, a multi-center study would likely be needed, ideally evaluating children with moderate and severe TBIs separately. When evaluating those with severe TBI due to AHT alone, there may be alterations in cerebral hemodynamics that we were unable to fully evaluate in this study. Furthermore, there may be factors outside the neurovascular response to injury, such as a combination of traumatic and asphyxia injury that occur in children with AHT that contribute to their significant and increased morbidity and mortality compared with trauma from other etiologies. One could hypothesize that the significant difference in seizure incidence in our cohort, consistent with prior studies, may suggest that seizure burden may have a significant role in outcome [8].

Our study has several limitations. This single-center retrospective study had a small sample size, which limited our ability to detect a significant difference between categories. TCD was performed once daily while continuous monitoring, though more resource intensive, may provide additional insight. Future studies should further evaluate the acute cerebral hemodynamics in children with AHT in a larger sample size and consider the use of continuous TCD monitoring as it may show individual variability with both $V_{\mathrm{MCA}}$ and autoregulation on an hour by hour basis not captured by intermittent monitoring. In addition, we compared children with AHT and a median age of approximately 4 months with children without AHT with a median age of 10 years. Only three children in the non-AHT group were under 2 years of age. We attempted to control for this by standardizing each patient's $V_{\text {MCA }}$ data to that expected for a child of similar age, gender, and the presence/absence of invasive mechanical ventilation.
Table 4 Percentage of studies with intact cerebral autoregulation $($ THRR $\geq 1.035)$

\begin{tabular}{lcccccccc}
\hline & Day 1 & Day 2 & Day 3 & Day 4 & Day 5 & Day 6 & Day 7 & Day 8 \\
\hline AHT total $(n=15)$ & $33 \%$ & $50 \%$ & $27 \%$ & $50 \%$ & $60 \%$ & $50 \%$ & $50 \%$ & $50 \%$ \\
AHT favorable $(n=7)$ & $25 \%$ & $50 \%$ & $25 \%$ & $50 \%$ & $100 \%$ & $50 \%$ & $100 \%$ & $50 \%$ \\
AHT unfavorable $(n=8)$ & $40 \%$ & $33 \%$ & $29 \%$ & $50 \%$ & $33 \%$ & $50 \%$ & $100 \%$ & $\mathrm{n} / \mathrm{a}$ \\
Non-AHT $(n=54)$ & $50 \%$ & $46 \%$ & $58 \%$ & $59 \%$ & $53 \%$ & $63 \%$ & $36 \%$ & $56 \%$ \\
$p$ value AHT versus Non-AHT & & & \multicolumn{7}{c}{0.4345} & & & \\
$p$ value AHT and outcome & & & & 0.5607 & & \\
\hline
\end{tabular}

$A H T$, abusive head trauma; non-AHT, non-abusive head trauma 
Future studies should compare children under 2 years with and without AHT to ensure that age alone does not negate specific changes to the neurovasculature in this population. Lastly, we were limited to short-term neurologic outcome data, and longer follow-up would likely be more informative.

\section{Conclusions}

In a small study of children with AHT, there were no statistically significant differences in $V_{\mathrm{MCA}}$ or percentage of extreme CBFV compared with those without AHT. When comparing children with AHT based on neurologic outcome, there was no difference in flow velocity in the MCA or basilar artery. There was no difference in the percentage of studies with intact autoregulation based upon the presence or absence of AHT, or neurologic outcome within the AHT group. Future studies with a larger sample size are needed to further evaluate the TCD findings in children with AHT, with an aim to elucidate the contribution of chronic head injury on the acute hemodynamics.

\section{Compliance with ethical standards}

The parent study was approved by the institutional review board and informed consent was obtained prior to patient enrollment.

Conflict of interest On behalf of all authors, the corresponding author states that there is no conflict of interest.

\section{References}

1. Aaslid R, Markwalder TM, Nornes H (1982) Noninvasive transcranial Doppler ultrasound recording of flow velocity in basal cerebral arteries. J Neurosurg 57:769-774. https://doi.org/10.3171/jns.1982. 57.6.0769

2. Beers SR, Wisniewski SR, Garcia-Filion P, Tian Y, Hahner T, Berger RP, Bell MJ, Adelson PD (2012) Validity of a pediatric version of the Glasgow Outcome Scale-Extended. J Neurotrauma 29:1126-1139. https://doi.org/10.1089/neu.2011.2272

3. Bode H, Wais U (1988) Age dependence of flow velocities in basal cerebral arteries. Arch Dis Child 63:606-611

4. Boop S, Axente M, Weatherford B, Klimo P Jr (2016) Abusive head trauma: an epidemiological and cost analysis. J Neurosurg Pediatr 18:542-549. https://doi.org/10.3171/2016.1.PEDS15583

5. Buckley EM, Miller BF, Golinski JM, Sadeghian H, McAllister LM, Vangel M, Ayata C, Meehan WP 3rd, Franceschini MA, Whalen MJ (2015) Decreased microvascular cerebral blood flow assessed by diffuse correlation spectroscopy after repetitive concussions in mice. Journal of cerebral blood flow and metabolism : official journal of the International Society of Cerebral Blood Flow and Metabolism 35:1995-2000. https://doi.org/10.1038/ jcbfm.2015.161

6. Choudhary AK, Servaes S, Slovis TL, Palusci VJ, Hedlund GL, Narang SK, Moreno JA, Dias MS, Christian CW, Nelson MD Jr, Silvera VM, Palasis S, Raissaki M, Rossi A, Offiah AC (2018) Consensus statement on abusive head trauma in infants and young children. Pediatr Radiol 48:1048-1065. https://doi.org/10.1007/ s00247-018-4149-1

7. Deans KJ, Minneci PC, Lowell W, Groner JI (2013) Increased morbidity and mortality of traumatic brain injury in victims of nonaccidental trauma. J Trauma Acute Care Surg 75:157-160

8. Greiner MV, Greiner HM, Care MM, Owens D, Shapiro R, Holland $\mathrm{K}$ (2015) Adding insult to injury: nonconvulsive seizures in abusive head trauma. J Child Neurol 30:1778-1784. https://doi.org/10. 1177/0883073815580285

9. Holmes JF, Palchak MJ, MacFarlane T, Kuppermann N (2005) Performance of the pediatric Glasgow coma scale in children with blunt head trauma. Acad Emerg Med Off J Soc Acad Emerg Med 12:814-819. https://doi.org/10.1197/j.aem.2005.04.019

10. Kochanek PM, Carney N, Adelson PD, Ashwal S, Bell MJ, Bratton S, Carson S, Chesnut RM, Ghajar J, Goldstein B, Grant GA, Kissoon N, Peterson K, Selden NR, Tasker RC, Tong KA, Vavilala MS, Wainwright MS, Warden CR, American Academy of Pediatrics-Section on Neurological S, American Association of Neurological Surgeons/Congress of Neurological S, Child Neurology S, European Society of P, Neonatal Intensive C, Neurocritical Care S, Pediatric Neurocritical Care Research G, Society of Critical Care M, Paediatric Intensive Care Society UK, Society for Neuroscience in A, Critical C, World Federation of Pediatric I, Critical Care S (2012) Guidelines for the acute medical management of severe traumatic brain injury in infants, children, and adolescents - second edition. Pediatr Crit Care Med 13(Suppl 1):S1-S82. https://doi.org/10.1097/PCC.0b013e31823f435c

11. LaRovere KL, O'Brien NF, Tasker RC (2016) Current opinion and use of transcranial Doppler ultrasonography in traumatic brain injury in the pediatric intensive care unit. J Neurotrauma 33:21052114. https://doi.org/10.1089/neu.2015.4344

12. Lindegaard KF, Nornes H, Bakke SJ, Sorteberg W, Nakstad P (1989) Cerebral vasospasm diagnosis by means of angiography and blood velocity measurements. Acta Neurochir 100:12-24

13. Lovett ME, Maa T, Chung MG, O'Brien NF (2018) Cerebral blood flow velocity and autoregulation in paediatric patients following a global hypoxic-ischaemic insult. Resuscitation 126:191-196. https://doi.org/10.1016/j.resuscitation.2018.02.005

14. Miller Ferguson N, Sarnaik A, Miles D, Shafi N, Peters MJ, Truemper E, Vavilala MS, Bell MJ, Wisniewski SR, Luther JF, Hartman AL, Kochanek PM, Investigators of the A, Decisions in Acute Pediatric Traumatic Brain Injury T (2017) Abusive head trauma and mortality-an analysis from an international comparative effectiveness study of children with severe traumatic brain injury. Crit Care Med 45:1398-1407. https://doi.org/10.1097/CCM. 0000000000002378

15. Nuno M, Ugiliweneza B, Zepeda V, Anderson JE, Coulter K, Magana JN, Drazin D, Boakye M (2018) Long-term impact of abusive head trauma in young children. Child Abuse Negl 85:3946. https://doi.org/10.1016/j.chiabu.2018.08.011

16. O'Brien NF (2015) Reference values for cerebral blood flow velocities in critically ill, sedated children. Childs Nerv Syst 31:22692276. https://doi.org/10.1007/s00381-015-2873-5

17. O'Brien NF, Maa T, Yeates KO (2015) The epidemiology of vasospasm in children with moderate-to-severe traumatic brain injury. Crit Care Med 43:674-685. https://doi.org/10.1097/CCM. 0000000000000745

18. Orru E, Huisman T, Izbudak I (2018) Prevalence, patterns, and clinical relevance of hypoxic-ischemic injuries in children exposed to abusive head trauma. Journal of neuroimaging : official journal of the American Society of Neuroimaging 28:608-614. https://doi. org/10.1111/jon. 12555

19. Parks S, Annest J, Hill H, Karch D (2012) Pediatric abusive head trauma: recommended definitions for public health surveillance and research. Centers for Disease Control and Prevention 
20. Smielewski P, Czosnyka M, Kirkpatrick P, McEroy H, Rutkowska H, Pickard JD (1996) Assessment of cerebral autoregulation using carotid artery compression. Stroke 27:2197-2203

21. Smielewski P, Czosnyka M, Kirkpatrick P, Pickard JD (1997) Evaluation of the transient hyperemic response test in headinjured patients. J Neurosurg 86:773-778. https://doi.org/10.3171/ jns.1997.86.5.0773

22. Thibeault CM, Thorpe S, O'Brien MJ, Canac N, Ranjbaran M, Patanam I, Sarraf A, LeVangie J, Scalzo F, Wilk SJ, Diaz-Arrastia R, Hamilton RB (2018) A cross-sectional study on cerebral hemodynamics after mild traumatic brain injury in a pediatric population. Front Neurol 9:200. https://doi.org/10.3389/fneur.2018.00200

23. van Santbrink H, Schouten JW, Steyerberg EW, Avezaat CJ, Maas AI (2002) Serial transcranial Doppler measurements in traumatic brain injury with special focus on the early posttraumatic period. Acta Neurochir 144:1141-1149. https://doi.org/10.1007/s00701002-1012-8

24. Wright JN (2017) CNS injuries in abusive head trauma. AJR Am J Roentgenol 208:991-1001. https://doi.org/10.2214/AJR.16.17602

25. Ziegler D, Cravens G, Poche G, Gandhi R, Tellez M (2017) Use of transcranial Doppler in patients with severe traumatic brain injuries. J Neurotrauma 34:121-127. https://doi.org/10.1089/neu.2015.3967

Publisher's note Springer Nature remains neutral with regard to jurisdictional claims in published maps and institutional affiliations. 